\title{
Linx
}

Revue des linguistes de l'université Paris X Nanterre

$61 \mid 2009$

Entre rection et incidence : des constructions verbales atypiques?

\section{La prosodie des verbes parenthétiques en français parlé}

Mathieu Avanzi

\section{CpenEdition}

Journals

Édition électronique

URL : http://journals.openedition.org/linx/1344

DOI : $10.4000 /$ linx. 1344

ISSN : 2118-9692

Éditeur

Presses universitaires de Paris Nanterre

Édition imprimée

Date de publication : 1 juin 2009

Pagination : 131-144

ISSN : 0246-8743

Référence électronique

Mathieu Avanzi, «La prosodie des verbes parenthétiques en français parlé », Linx [En ligne], 61 | 2009, mis en ligne le 01 juin 2013, consulté le 19 avril 2019. URL : http://journals.openedition.org/linx/1344 ; DOI : $10.4000 /$ linx. 1344 


\title{
La prosodie des verbes parenthétiques en français parlé
}

\author{
Mathieu Avanzi \\ Université de Neuchâtel
}

L'objectif de cette contribution est double ${ }^{1}$ : il s'agit d'une part de vérifier l'hypothèse selon laquelle la prosodie joue le rôle de «subordonnant» dans les contextes où le morphème qu- n'est pas exprimé (je crois Ø il va venir); d'autre part de discuter des modélisations qui font des verbes parenthétiques des éléments prosodiquement séparés de la structure hôte lorsqu'ils ont le statut d'incise médiane (il va je crois venir) ou d'incise finale (il va venir je crois). Pour chacune des trois positions (initiale, médiane et finale), je rappelle d'abord quelles sont les hypothèses formulées dans les travaux de mes prédécesseurs, et commente ensuite les résultats obtenus avec l'analyse de données authentiques. Je conclus enfin sur les conséquences que les résultats ont sur notre connaissance de l'interface prosodie/syntaxe. Avant de passer à la description à proprement parler, je présente les corpus de français parlé dans lesquels j’ai recherché les exemples de verbes parenthétiques analysés dans cette étude, ainsi que le traitement qui a été appliqué en vue de leur analyse prosodique.

\section{Présentation des données}

Les énoncés sur lesquels se base le commentaire sont extraits de corpus de parole « spontanée $»^{2}$, à dominante monologique. Les locuteurs sont tous des francophones natifs, originaires de la région parisienne (CFPP2000, Branca et al. 2009), de la région

\footnotetext{
${ }^{1}$ Cet article reprend sous une forme synthétique les conclusions obtenues dans travaux antérieurs consacrés à la prosodie des verbes dits parenthétiques ( $c$. Avanzi \& Glikman ici-même) en français parlé (Avanzi 2012; Gachet \& Avanzi 2011).

${ }^{2}$ La notion de parlé «spontané » est problématique pour les enregistrements réalisés à micro ouvert (Bilger \& Blanche-Benveniste 1999). Je m’en sers ici pour désigner de la parole non lue non préparée.
} 
PACA (CID, Bertrand et al. 2008), de l'ensemble de la métropole (CRFP, DELIC 2004 ; PFC, Durand et al. 2005), ou de Belgique francophone (VALIBEL, Dister et al. 2010). Les formes verbales recherchées automatiquement sont celles qui ont été identifiées comme les plus fréquentes par Blanche-Benveniste \& Willems (2007) et Willems \& Blanche-Benveniste (2010), soit, dans l'ordre décroissant : je crois, je pense, je trouve, il paraît/parait-il, il me semble/ me semble-t-iß .

L'ensemble des énoncés retenus pour l'analyse a ensuite été transcrit et aligné automatiquement en phonèmes, syllabes et mots graphiques dans Praat (Boersma \& Weeninck 2011) avec le script Easyalign (Goldman 2011). Lors de la phase de correction manuelle, j’ai indiqué pour chacun des énoncés quelles étaient les syllabes associées une disfluence (hésitation, faux-départ, interruption, etc.), afin de ne pas gêner les mesures automatiques des variations prosodiques relatives finalement conduites dans Analor (Avanzi et al. 2010, 2011 ; Avanzi 2012 : chap. 3). Sur la base de l'appréciation des variations de paramètres classiques tels que la f0, la durée et la présence des pauses, ce logiciel permet de procéder à une détection automatique des proéminences accentuelles et d'attribuer ensuite à chacune des syllabes identifiées comme saillantes un score qui indique son degré de force relative (entre $0 / 10$ et 10/10) $)^{4}$. Les valeurs des seuils utilisés pour le traitement des exemples présentés dans les annexes de cet article ont été optimisées à partir d'un entraînement sur un corpus codé manuellement en amont. Je ne reviens pas ici sur les détails de fonctionnement de l'algorithme, et me permets de renvoyer le lecteur intéressé aux travaux mentionnés ci-dessus, dans lesquels il est largement présenté.

\section{Position initiale}

2.1. Le premier contexte étudié concerne des énoncés dans lesquels le verbe parenthétique précède une construction verbale qu'il mitige, sans être suivi du relateur $q u$ - Les exemples (1)-(5) illustrent ce dont il est question:

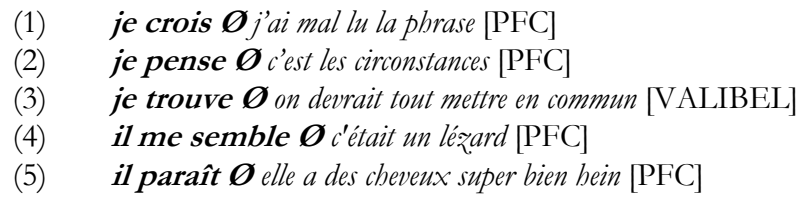

L'absence de relateur après les verbes parenthétiques, attestée en ancien et moyen français (Glikman 2009), est donnée comme impossible en français moderne (Blanche-Benveniste 1989; Apothéloz 2003; Blanche-Benveniste \& Willems 2007), bien qu'elle ait été constatée par des auteurs ayant écrit sur le français populaire (Bauche 1920 ; Gadet 1992), sur les variétés de français parlées au Québec (Martineau 1993), en Afrique (Akissi Boutin 2007) ou en Guadeloupe (Putska 2010.). Sans doute la fréquence de ce phénomène est moins importante en français « européen » que dans les conversations anglo-saxonnes (où l'omission du relateur «est la norme", selon Biber et al., 1999, p. 681). Quoiqu'il en soit, l'examen des contextes larges ainsi que des sonagrammes des extraits (1)-(5) confirme que l'omission de qu-en français moderne

3 J'ai laissé de côté les formes impliquant les verbes dire comme je dirais, on dirait.

${ }^{4}$ Les résultats de ces analyses peuvent être visualisés sur les copies d'écran disposées en annexes. 
est tout à fait possible. A titre d'illustration, je commente l'exemple (1) supra, que je redonne ci-dessous dans son contexte :

(6) la côte escarpée du mont Saint-Pierre connaît des barrages chaque fois que des opposants de tous les bords mani- je crois j'ai mal lu la phrase (rires) manifestent leur colère [PFC]

Il est impossible de dire ici que je crois porte sur ce qui précède plutôt que sur ce qui suit. La locutrice est en train de lire un texte, et s'interrompt après s'être rendu compte qu'elle a mal prononcé un segment de la phrase. Il ne s'agit pas d'une bribe, et l'examen du spectrogramme ( $c$. figure 1$)^{5}$ montre bien qu'aucune occlusive n'est produite après je crois. Si elle l'était, on observerait dans cette position la même marque verticale que l'on observe pour le $[\kappa]$ initial de croire.

2.2. Lorsque le lien entre les deux propositions n'est pas indiqué par un relateur segmental, l'hypothèse formulée par divers auteurs est que la prosodie joue le rôle de morphème «subordonnant » :

La marque morphologique des conjonctions de subordination, les «subordonnants》 [peuvent] être tout aussi bien d'ordre sémantique ou éventuellement prosodique. (Andersen, 1997, p. 82)

Au plan de la substance, le verbe parenthétique et la construction qui le suit seraient prononcés de façon liée, sans interruption :

En règle générale, on peut dire qu'une même courbe intonative signifie qu'il y a intégration de la proposition au reste de l'énoncé, donc subordination ou implication (Andersen, 1999, p. 70)

2.3. L'analyse des exemples confirme que le patron prosodique des couplages de constructions verbales tels que (1)-(5) est toujours «lié », i.e. sans rupture intonative interne. Ainsi, dans l'exemple de la figure 2, la proéminence accentuelle finale dont est assorti le verbe parenthétique (je crois) est trop faible pour créer un sentiment de détachement à l'écoute : comparée à la proéminence finale de l'énoncé (j'ai mal lu la phrase), elle ne fait pas l'objet d'un écart mélodique très important, ni d'un allongement significatif.

Si la frontière dont était assorti le verbe parenthétique ne se distingue pas vraiment, en termes perceptifs, de la frontière finale de la proposition à laquelle il s'accole, on observe une ambiguité de rattachement entre le verbe parenthétique et son contexte. Le verbe il me semble de la figure 3 donne une illustration de ce dont il s'agit. La proéminence accentuelle qui ponctue le segment il me semble ne se distingue pas vraiment de la proéminence finale du segment qui la précède (ily a deux ans), ni de celle qui la suit (ils ont encore livrế). A l'écoute, on ne sait pas, avec une telle prosodie, si le verbe parenthétique doit être analysé comme se rapportant à ce qui suit ou à ce qui précède.

2.4. L'absence de qu-après les verbes parenthétiques lorsqu'ils (pseudo-)régissent ${ }^{6}$ une complétive est bien palliée par une prosodie particulière. Ces complexes sont actualisés par des patrons mélodiques de type «englobant» : le verbe parenthétique et la construction verbale à laquelle il se rapporte forment un seul et même paquet intonatif de rang supérieur. Si la prosodie ne prend pas le relais lorsque les marques

\footnotetext{
${ }^{5}$ Pour des raisons de mise en page, les figures sont placés en annexes à la fin de l'article.

${ }^{6}$ J'utilise le préfixe "pseudo" entre parenthèses pour rendre compte du fait que la syntaxe des verbes faibles pose encore problème pour l'analyste, $c f$. Gachet (ici-même).
} 
Mathieu Avanzi

segmentales font défaut, i.e. que le verbe parenthétique n'est pas explicitement inclus dans le même paquet prosodique que la proposition qu'il suit, il est interprété comme un élément flottant, ou disjoint : il ne porte pas plus sur ce qui précède que sur ce qui suit.

\section{Position médiane}

3.1. Les verbes parenthétiques en position médiane peuvent intervenir à peu près n'importe où dans l'énoncé (Marandin 1999), mais c'est entre deux constituants syntaxiques majeurs qu'on les rencontre le plus fréquemment en français parlé :

(7) Son père à lui a été eub élevé je crois à Paris [PFC]

(8) il a été fait euh je pense l'année dernière [PFC]

(9) pis eub intellectuellement c'est quand même plus enrichissant je trouve d'échanger avec d'autres [PFC]

(10) elle doit finir de passer son deuxième degré il me semble cette année [PFC]

(11) beaucoup de gens paraît-il ont changé ont voulu eub devenir meilleurs [PFC]

3.2. Les incises en position médiane ont longtemps été décrites comme des éléments «hors énoncé » sur le plan prosodique, se démarquant explicitement de la structure hôte en raison de la présence de frontières intonatives accusées avant et après (montée de f0, allongements, pauses), de leur registre et/ou de leur débit (cf. Gachet \& Avanzi 2008 ; Dehé 2009 pour des états de la question) :

L'insertion entraîne une interruption qui se répercute fortement sur la ligne mélodique et qui se marque à l'oral par une intonation parenthétique et éventuellement par une pause [Riegel et al., 2009, p. 769]

Le débit dans l'incise serait régulièrement plus rapide que dans les syntagmes accompagnant, l'intensité dans l'incise serait plus faible que dans le reste de la phrase [Wunderli, 1988, p. 262]

Certaines études ont cependant montré qu’il était rare que le verbe parenthétique soit séparé de ce qui précède et de ce qui suit par des pauses silencieuses. En général, la pause est réalisée soit avant soit après le constituant parenthétique, l'autre frontière étant marquée uniquement par un mouvement mélodique majeur et/ou un allongement de durée. Dans l'exemple de la figure 4, la pause silencieuse intervient entre le segment parenthétique et ce qui le suit (je crois \# plusieurs appartements) le constituant précédant se termine par une montée mélodique à valeur continuative (moi j'achèterais $\underline{\text { même }}$.

Or, comme l'a montré Delais-Roussarie (2008), il arrive que la frontière dont est assorti le constituant à gauche de l'incident ne soit pas être réalisée phonétiquement. Une telle situation se produit essentiellement lorsque le parenthétique intervient après un morphème grammatical, qui n'est pas attracteur d'accent final (Garde 1965). Dans l'exemple de la figure 5, le segment je pense intervient après le relateur qui, qui n'est pas accentogène. Le bord droit du parenthétique est en revanche marqué par une montée mélodique et un allongement syllabique final. De ce point de vue, il constitue un groupe intonatif différent de ce qui le suit

3.3. La prise en compte de données authentiques montre que si ces deux réalisations sont bien attestées, il en existe d'autres. L'examen de la figure 6 atteste ainsi 
d'un verbe parenthétique séparé de sa base par une frontière marquée à droite, mais pas à gauche. L'allongement et la montée mélodique assortissant le groupe des gros propréétaires terriens lui confèrent le statut de syntagme intonatif. Rien de tel n'est perceptible sur le bord du verbe parenthétique (je crois), qui forme avec l'adjectif qui le suit (immobiliers) un seul et même groupe prosodique. Quant à l'exemple sous la figure 7, il atteste d'un parenthétique qui ne se démarque pas prosodiquement de la construction hôte ni par son contexte gauche, ni par son contexte droit. On ne perçoit pas de rupture prosodique entre c'est qui fait un peu, je pense et la renommée eub : les trois segments sont prononcés dans le même groupe intonatif.

3.4. Les raisons de ces réalisations qui remettent en cause les modèles formels de l'interface prosodie/syntaxe sont multiples. D'un point de vue métrique, les incises sont des segments relativement légers, souvent monosyllabiques, ce qui explique qu'ils peuvent s'affixer facilement sur le plan intonatif avec des éléments de leur contexte. D'un point de vue discursif, on peut arguer que cette tendance à se fondre dans le même syntagme intonatif que les parties de l'élément qui les attire est une trace d'optimisation du travail communicatif, à l'origine de processus de réanalyses potentielles du verbe parenthétique en marqueur de discours.

\section{Position finale}

4.1. La troisième position que les verbes parenthétiques peuvent occuper dans l'énoncé est une position finale, après le noyau. Soit les exemples :

(12) l'autre avait de l'argent je crois [PFC]

(13) ça été fondamental je pense [CFPP]

(14) ça a augmenté énormément je trouve [CFPP]

(15) c'est le quartier Fécand il me semble [CFPP]

(16) c'est fort saccadé il me semble [VALIBEL]

4.2. Comme pour la position médiane, les incises en position finale sont décrites comme formant des syntagmes intonatifs distincts de la séquence de base, qui est assorti de la frontière terminale de l'énoncé, frontière par définition la plus forte et que rien ne peut dominer :

Les incidents sont prosodiquement autonomes; optionnellement, ils sont séparés du reste de la phrase (...) par une pause ou un allongement de la dernière syllabe, un contraste de FO sur la dernière syllabe, ou un changement global de registre (Bonami \& Godard, 2008, p. 2408)

L'exemple de la figure 8 fournit une illustration prototypique de l'incise en position finale, telle qu'elle est généralement décrite dans la littérature. La fin de la proposition on le garde est actualisée par un contour intonatif descendant au niveau infra-grave, et le verbe parenthétique en incise $j e$ pense est prononcée sur un registre bas et plat, sans modulation. Bien entendu, d'autres réalisations sont possibles, lesquelles dépendent de la visée communicative (qui détermine la forme du contour terminal de la première proposition) et de la posture interactionnelle adoptée par le sujet parlant (Berrendonner 2008). On trouve ainsi des incises copiant le contour montant de la séquence de base sur un registre plus haut (figure 9), ou doté d'un contour mélodique différent du contour de la construction matrice (figure 10). 
Mathieu Avanzi

4.3. Or, il n'est pas impossible de rencontrer des verbes parenthétiques en position finale qui ne sont pas précédés d'une frontière majeure à caractère terminal.

Le tracé intonatif de la figure 11 permet de voir que si certaines incises sont bien précédées d'une frontière, celle-ci n'a forcément le statut d'une frontière terminale. Le bord droit du segment qui a été construit dans les années dix-buit cent trente ne porte pas la proéminence la plus forte de l'énoncé. C'est la syllabe finale de l'incise qui a cette propriété. On voit en effet qu'elle est assortie d'un contour continuatif supérieur, dont la valeur interactionnelle est celle d'une demande de feedback (Portes et al. 2007).

Quant au tracé intonatif de la figure 12, il atteste d'une incise qui n'est pas précédée d'une frontière prosodique quelconque. Dans cet exemple, l'effacement de frontière est total: on ne perçoit aucune variation prosodique entre la construction matrice y a moins de choses moins d'activités et l'incise je pense. Les deux segments ne forment qu'un seul et même groupe intonatif.

4.4. Comme les autres éléments relevant du paradigme des incises finales, le contour des verbes recteurs parenthétiques peut dominer celui de l'énoncé principal, voir le remplacer. On expliquera dans ce cas comme dans le précédent $(\$ 3.3)$ que ces affaiblissements de frontière sont des traces d'une optimisation du travail communicatif, à l'origine de réanalyses syntaxiques du verbe parenthétique comme un marqueur discursif. Des études sur la prosodie des marqueurs discursifs reconnus comme tels devraient permettre de confirmer cette hypothèse.

\section{Conclusion}

Cette note de synthèse avait pour objectif de présenter avec des exemples nouveaux les conclusions obtenues dans des travaux antérieurs, portant sur la prosodie des verbes parenthétiques. J'ai montré que lorsque le verbe parenthétique en position initiale n'était pas suivi de qu-, une prosodie particulière était effectivement de rigueur, les propriétés sémantiques du parenthétique ne permettant pas de prédire sur la seule base du contexte qu'il se rapporte à ce qui suit. L'étude des verbes parenthétiques en positions médiane et finale m’a permis de rendre compte de phénomènes qui remettent en cause une conception classique de l'interface prosodie/syntaxe en français parlé, qui voudrait qu'aux frontières syntaxiques fortes correspondent des frontières prosodiques majeures. Les résultats de cette étude doivent être à présent complétés par des travaux sur la sémantique et la pragmatique des verbes parenthétiques, qui permettront de dire si les affaiblissements prosodiques constatés sont corrélés à un changement linguistique en cours, ou pas. 


\section{RÉFÉRENCES BIBLIOGRAPHIQUES}

Akissi Boutin, B. (2007), « De, que, pour, subordonnants, et variation en français », Linx, n57, pp. $57-68$.

Andersen, H. L. (1997), Propositions parenthétiques et subordination en français parlé, Thèse de doctorat, Université de Copenhague.

ApOThÉlOz, D. (2003), «Grammaticalisation ou différentiel de grammaticité ? Le cas de la rection dite 'faible' », Verbum, n³, pp. 241-262.

Avanzi, M. (2012, sous presse), L’interface prosodie/syntaxe en français parlé. Dislocations, incises, asyndètes, Bruxelles, Peter Lang.

Avanzi, M., LACHERET-Dujour, A. \& ViCTORRI, B. (2010), «A corpus-based learning method for prominence detection in spontaneous speech», Prosodic Prominence: Perceptual and Automatic Identification, Proceedings of Speech Prosody 2010 Workshop, Chicago, May 10.

Avanzi, M., LACHERET-Dujour, A., OBIN, N. \& VictorRi, B. (2011), « Vers une modélisation continue de la structure prosodique: le cas des proéminences syllabiques », Journal of French Language Studies, nº21/1, pp. 53-71.

AvanZI, M. \& GLIKMAn, J. (ici-même), « Présentation ».

BAuche, H. (1920), Le langage populaire, Paris, Payot.

BERRENDONNER, A. (2008), «Il est beau, le lavabo: il fait problème, cet intonème », in Birkelund, M., Mosegaard Hansen, M. \& Norén, C. (éds), L'énonciation dans tous ses états. Mélanges offerts à Henning Nolke à l'occasion de ses soixante ans, Bern, Peter Lang, pp. 669-687

Bertrand, R., Blache, P., Espesser, R., Ferré, G., Meunier, C., Priego-Valverde, B. \& RAuZY, S. (2008), « Le CID. Corpus of Interactional Data. Annotation et exploitation multimodale de parole conversationnelle », Traitement Automatique des Langues, n 49/3, pp. 1-30.

Biber, D., Johansson, S., LeECH, G., ConRAD, S. \& Finegan, E. (1999), The Longman Grammar of Spoken and Written English, New York, Longman.

BlanCHE-Benveniste, C. (1989), «Constructions verbales 'en incise' et rection faible des verbes », Recherches sur le français parlé, $\mathrm{n}^{\circ} 9$, pp. 53-73.

Bilger, M. \& Blanche-Benveniste, C. (1999), «Français parlé-oral spontané. Quelques réflexions ", Revue française de linguistique appliquée, $\mathrm{n}^{\circ} 4 / 2$, pp. 21-30.

Blanche-Benveniste, C. \& Willems, D. (2007), «Un nouveau regard sur les verbes faibles », Bulletin de la Société Linguistique de Paris, $\mathrm{n}^{\circ} 102 / 1$, pp. 217-254.

Boersma, P. \& WEENINK, D. (2011), Praat, version 2.6. www.praat.org.

Bonami, O. \& Godard, D. (2008), «Syntaxe des incises de citation», Actes du 1er Congrès Mondial de Linguistique Française, pp. 2395-2408.

Branca-Rosoff, S., Fleury, S., Lefeuvre, F. \& Pires, M. (2009), Discours sur la ville. Corpus de Français Parlé Parisien des années 2000 (CFPP2000), http://ed268.univ-paris3.fr/CFPP2000/

DEHÉ, N. (2009), «Clausal parentheticals, intonational phrasing, and prosodic theory », Journal of Linguistics, ${ }^{\circ} 45 / 3$, pp. 569-615. 
Mathieu Avanzi

DeLIC, (2004), « Présentation du Corpus de Référence du Français Parlé », RSFP, n 18, pp. 11-42.

Delais-Roussarie, (2008), "Structure prosodique et prosodie incidente », Verbum, n³0/1, pp. 37-52.

Dister, A., M. Francard, Ph. Hambye, P., \& Simon, A. C. (2009), « Du corpus à la banque de données. Du son, des textes et des métadonnées. L'évolution de banque de données textuelles orales VALIBEL (1989-2009)", Cahiers de l'Institut de Linguistique de Louvain, n³3/2, pp. 113-129.

Durand, J., LAKS, B. \& LyChe, Ch. (2005), «Un corpus numérisé pour la phonologie du français », in G. Williams (éd.) La linguistique de corpus, Rennes: Presses Universitaires, pp. 205-217. Voir aussi <http://www.projet-pfc.net/>

GACHET, F. (ici-même), « Les verbes parenthétiques : un statut syntaxique atypique ?».

GACHET, F. \& AvANZI, M. (2008), « La prosodie des parenthèses en français spontané », Verbum, n³0/1, pp. 53-84.

GACHET, F. \& AvANZI, M. (2011), "Description prosodique des 'recteurs faibles en incise' », in Yoo, H-Y \& Delais-Roussarie, E. (éds), Proceedings from IDP 2009, Paris, Septembre 2009, pp. 173-189. http://makino.linguist.jussieu.fr/idp09/actes en.htm.

GADET, F. (1992), Le français populaire, Paris, PUF.

Garde, P. (1965), L'accent, Paris, PUF.

Glikman, J. (2009), Parataxe et subordination en ancien français, Thèse de doctorat, Universités de Paris Ouest Nanterre et de Postdam.

Goldman, J.-P. (2011), « EasyAlign: an automatic phonetic alignment tool under Praat », Proc. Interspeech, pp. 3233-3236.

MARANDIN, J.-M. (1999), Grammaire de lincidence, manuscrit, < http://www.llf.cnrs.fr/fr/Marandin/>

Martineau, F. (1993), « Rection forte et rection faible des verbes : l'ellipse de que en français du Québec et de l'Ontario", in Birdsong, D. \& Montreuil, J.-P. (éds), Advances in Romance linguistics, pp. 275-287.

Portes, C., BERTRAND, R. \& EsPesser, R. (2007), « Contribution to a grammar of intonation in French. Form and function of three rising patterns ", Nouveaux Cabiers de linguistique française, $\mathrm{n}^{\circ} 28$, pp. 155-162.

PUTSKA, E. (2010). «La subordination sans subordonnant en français guadeloupéen - créolisme ou pseudo-créolisme », in Drescher, M. \& Neumann-Holzschuh, I. (éds), La syntaxe de l'oral dans les variétés non-hexagonales du français, Tübingen, Stauffenburg, pp. 149-166..

Riegel, M., PeLlat, J.-C. \& Rioul, R. (2009), Nowvelle Grammaire méthodique du français, Paris, PUF.

WiLlems, D. \& BLANCHE-Benveniste, C. (2010), «Verbes 'faibles' et verbes à valeur épistémique en français parlé : il me semble, il paraît, j’ai l'impression, on dirait, je dirais », in Iliescu, M. et al. (éds), Actes du XXVe Congrès International de Linguistique et de Philologie Romanes, Innsbruck, septembre 2007, Tome IV, De Gruyter, pp. 565-577.

Wunderli, P. (1988), «Compte rendu de l'ouvrage : Problèmes de prosodie, Léon, P. \& Rossi, M. (éds) », Cabiers Ferdinand de Saussure, n²42, pp. 251-266. 


\section{ANNEXES}

La figure 1 est une copie d'écran du logiciel Praat (Boersma \& Weenink 2011), les figures suivantes sont des copies d'écran du logiciel d'analyse prosodique Analor (Avanzi, Lacheret-Dujour \& Victorri 2010). L'évolution de la f0 (en traits noirs) peut être mesurée en demi-tons (en filigrane, la distance entre deux lignes fines vaut 1 demiton, la distance entre deux traits épais 4 demi-tons) ou en hertz (les valeurs numériques sont affichées sur la gauche); la durée des segments étiquetés est donnée en millisecondes au dessus de la bande dans laquelle évolue la courbe de f0. Les chiffres plus foncés au-dessus indiquent le temps en secondes du segment par rapport à l'enregistrement du fichier total. Les différentes couches d'alignement, importées directement depuis les fichiers d'alignement au format textgrid (Praat), sont affichées en dessous de cette bande, en l'occurrence, de haut en bas : les phonèmes et les syllabes en alphabet SAMPA, et la transcription des mots graphiques.

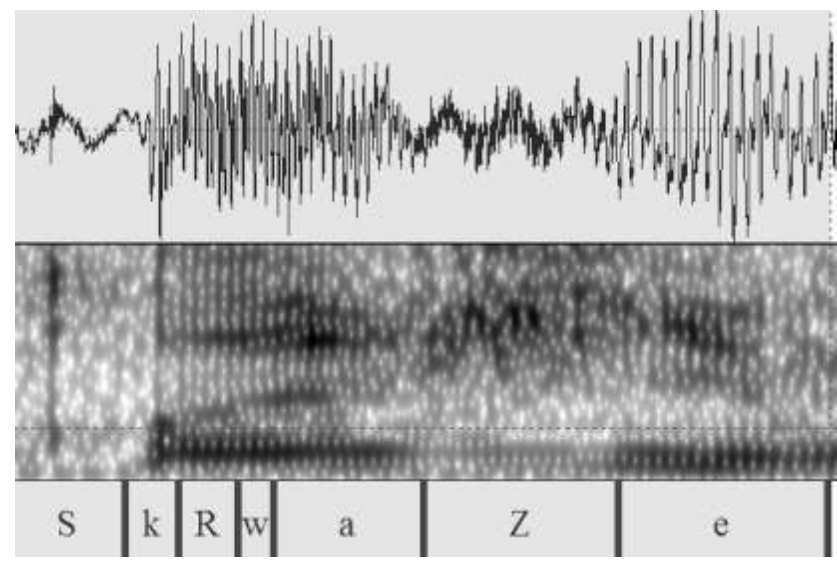

Figure 1. Copie d'écran Praat. Spectrogramme de la suite je crois j'ai [cf. ex. (1)]

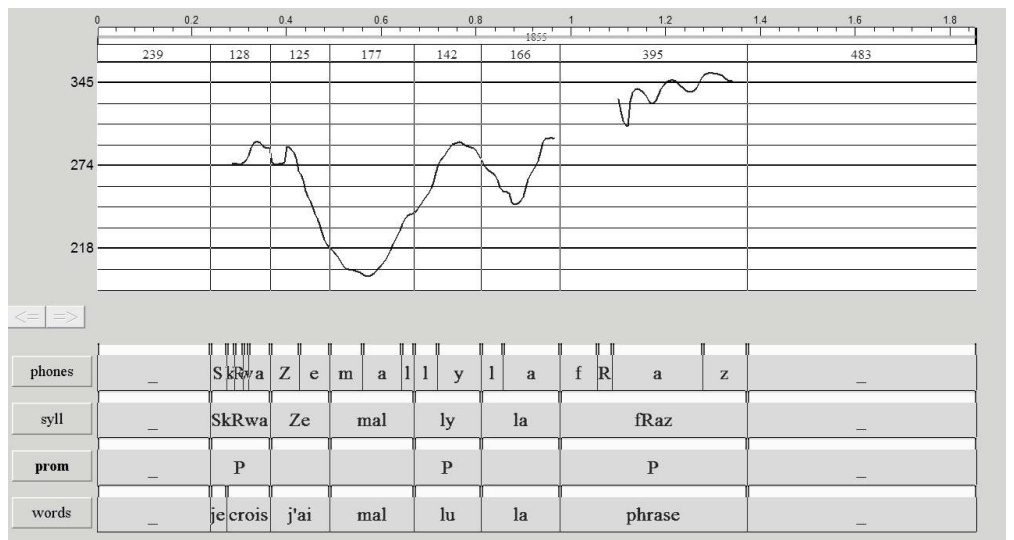

Figure 2. Copie d'écran Analor. Tracé intonatif de l'énoncé je crois j'ai mal lu la phrase [PFC] 


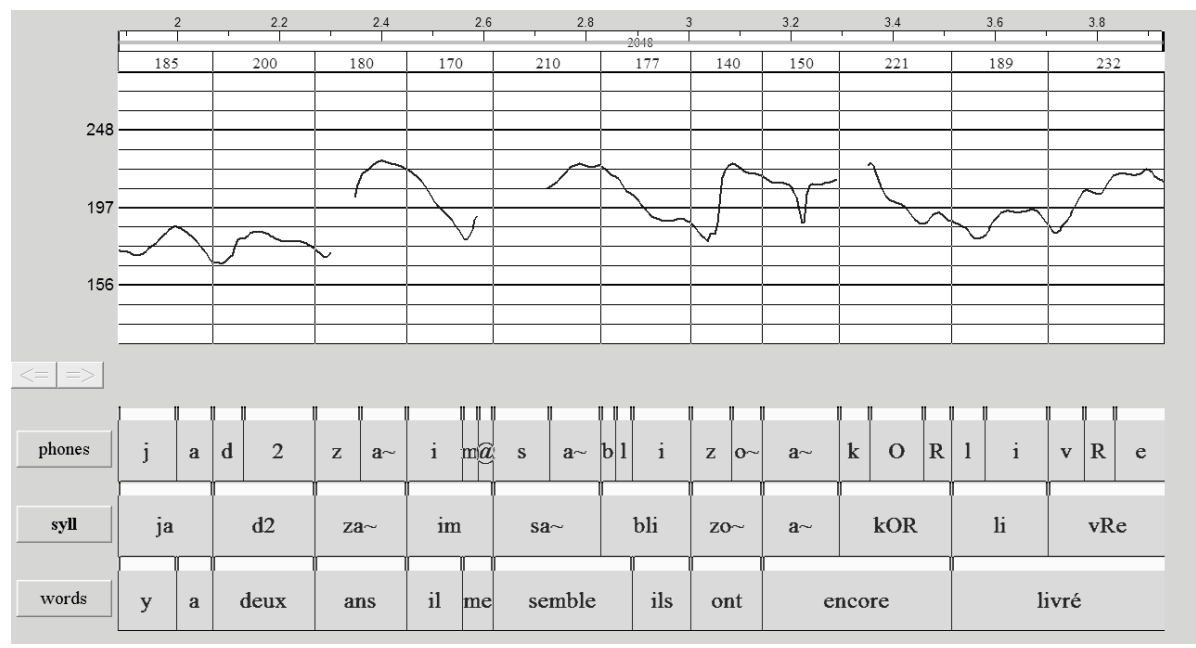

Figure 3. Copie d'écran Analor.

Tracé intonatif de l'énoncé il y a deux ans il me semble ils ont encore livré [PFC]

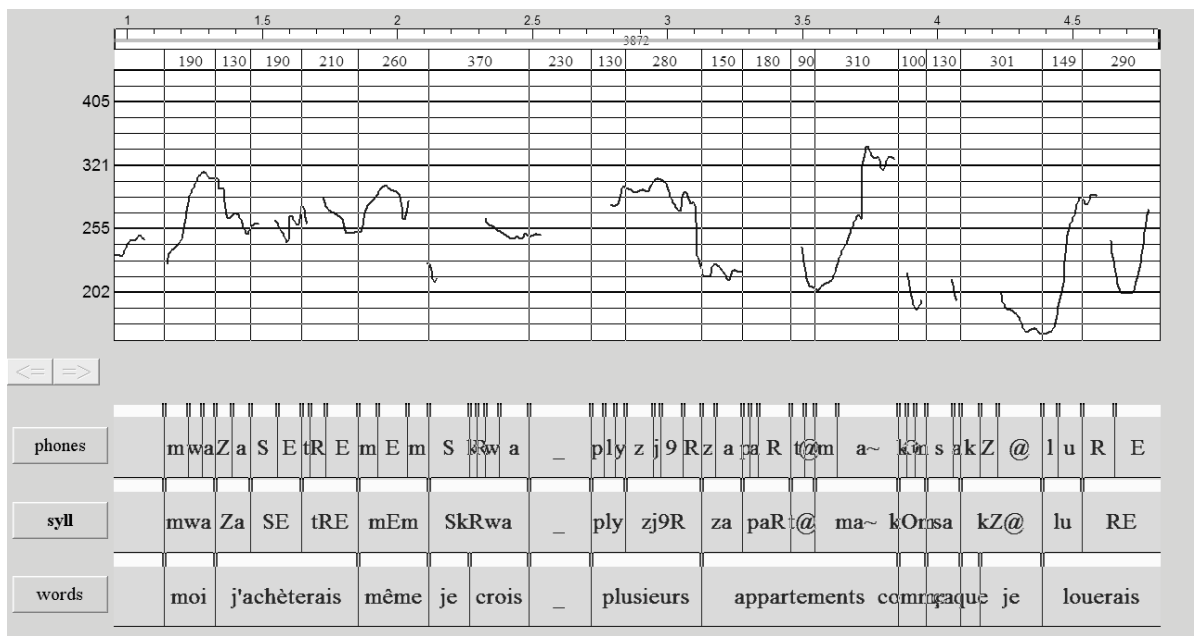

Figure 4. Copie d'écran Analor.

Tracé intonatif de l'énoncé moi j'achèterais même je crois plusieurs appartements que je louerais [PFC] 


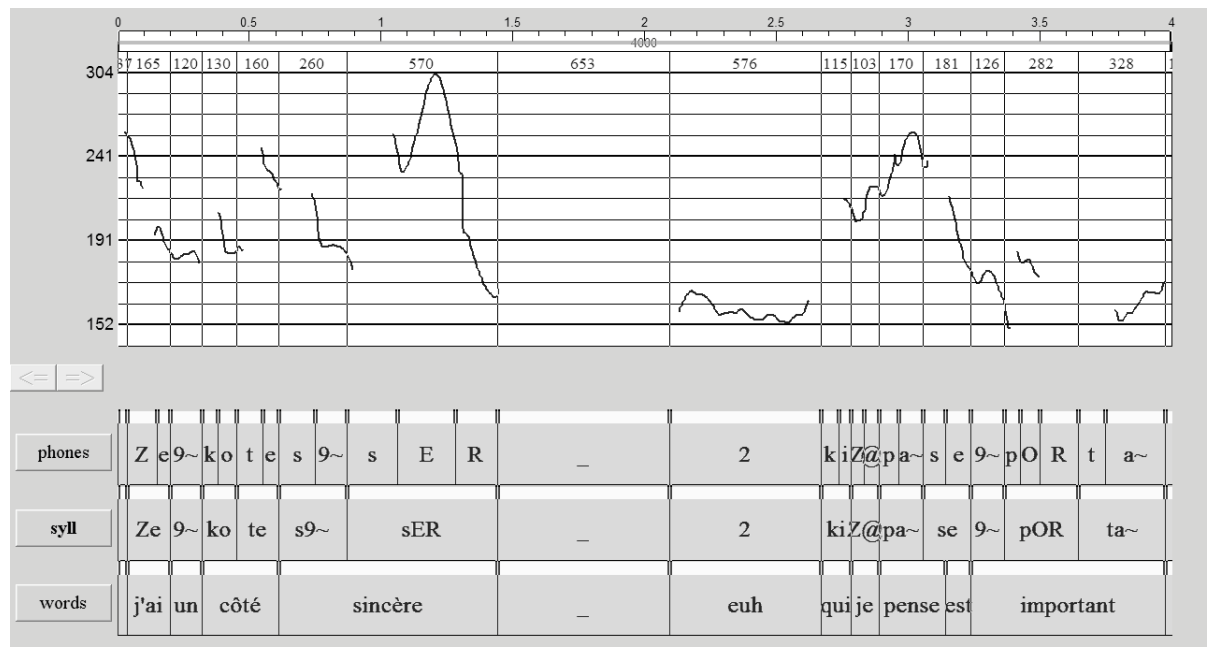

Figure 5. Copie d'écran Analor.

Tracé intonatif de l'énoncé $j$ 'ai un côté sincère - euh qui je pense est important [CRFP]

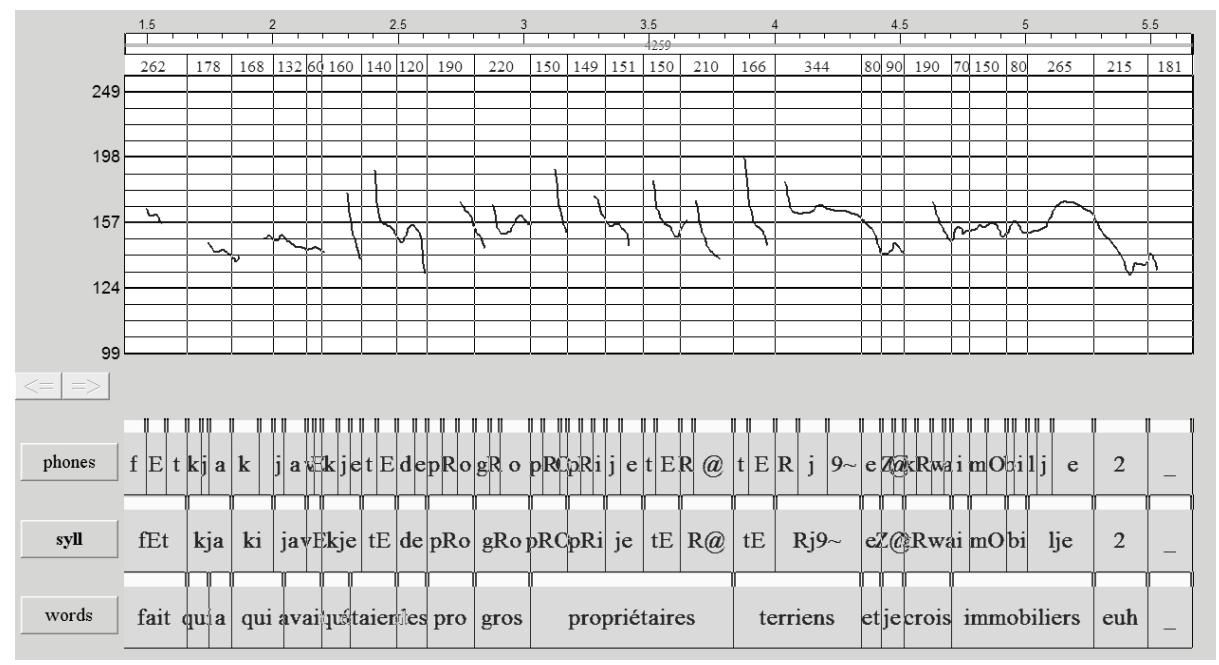

Figure 6. Copie d'écran Analor.

Tracé intonatif de l'énoncé qui avaient qui étaient des pro-gros propriétaires terriens et je crois immobiliers [PFC] 


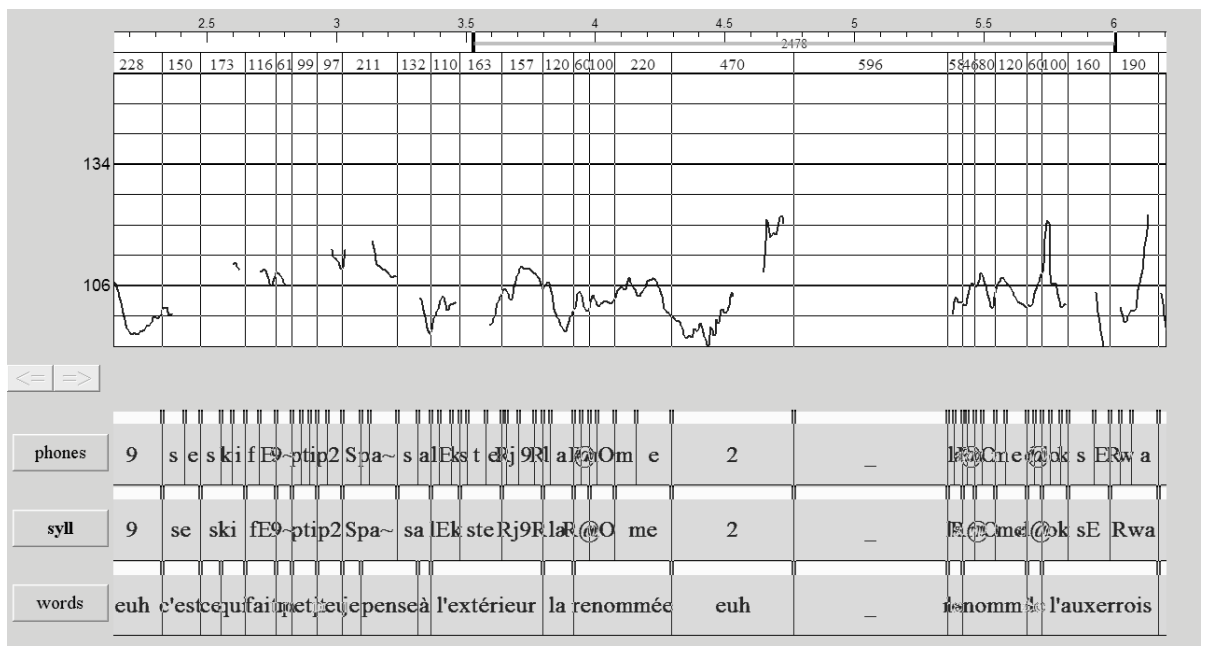

Figure 7. Copie d'écran Analor.

Tracé intonatif de l'énoncé eub c'est ce qui fait je pense à l'extérieur la renommée eub la renommée de l'Auxerrois [CRFP]

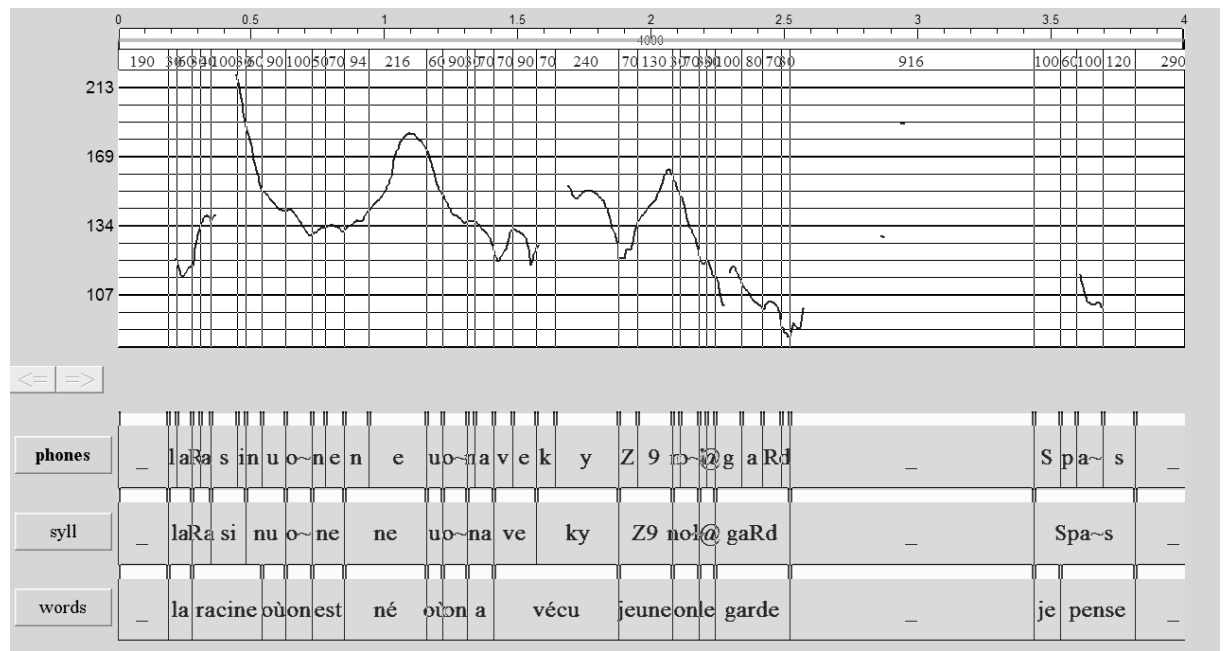

Figure 8. Copie d'écran Analor.

Tracé intonatif de l'énoncé la racine où on est né où on a vécu on le garde - je pense [CFPP2000] 


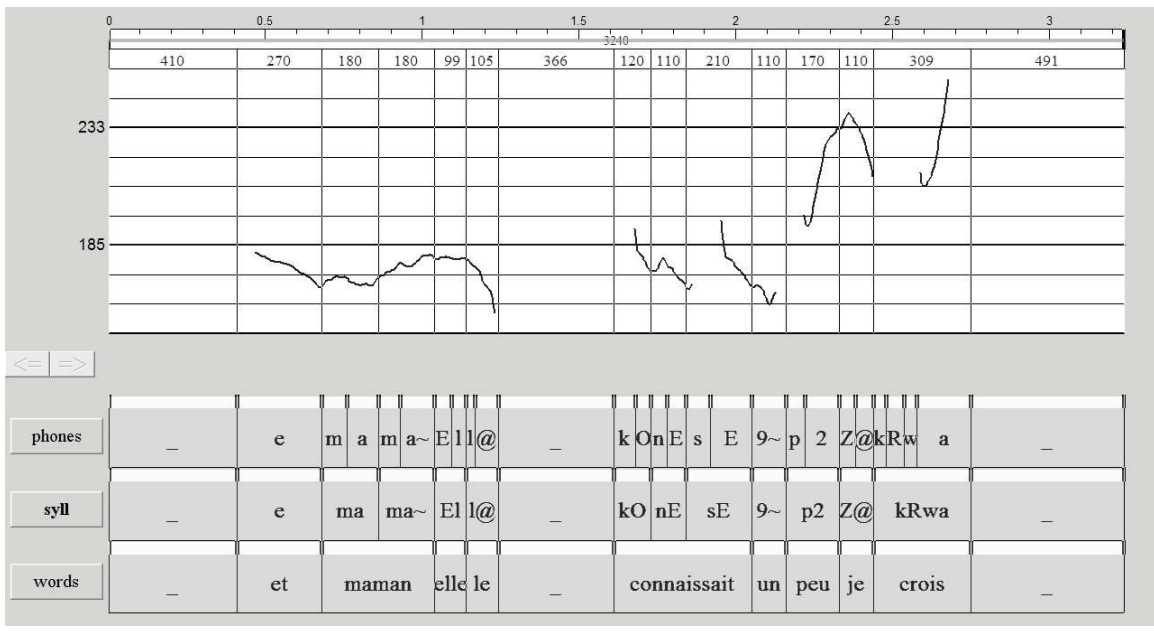

Figure 9. Copie d'écran Analor.

Tracé intonatif de l'énoncé et maman elle le - connaissait un peu je crois [PFC]

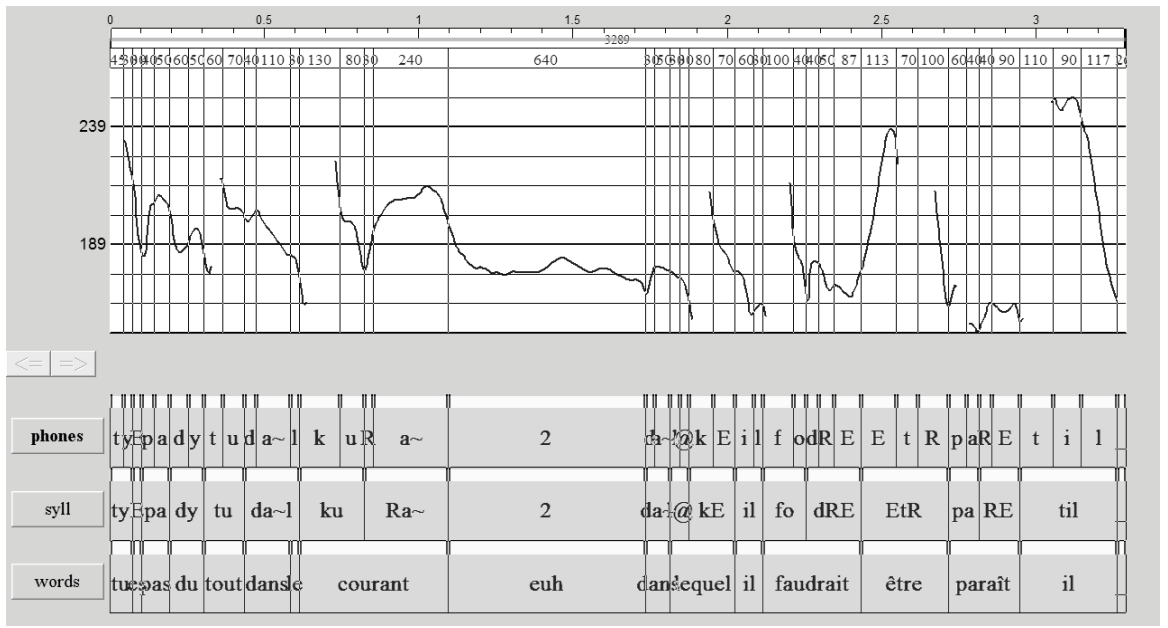

Figure 10. Copie d'écran Analor.

Tracé intonatif de l'énoncé tu es pas du tout dans le courant dans lequel il faudrait être paraît-il [CID] 


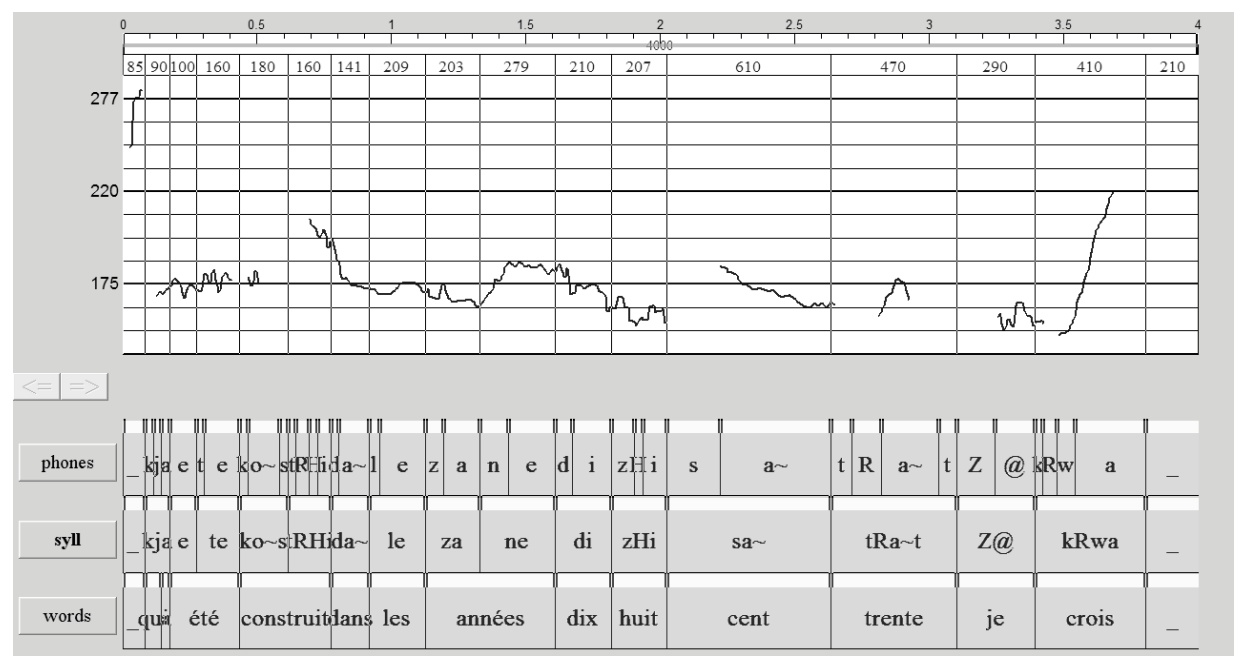

Figure 11. Copie d'écran Analor.

Tracé intonatif de l'énoncé qui a été construit dans les années dix-buit cent trente je crois [PFC]

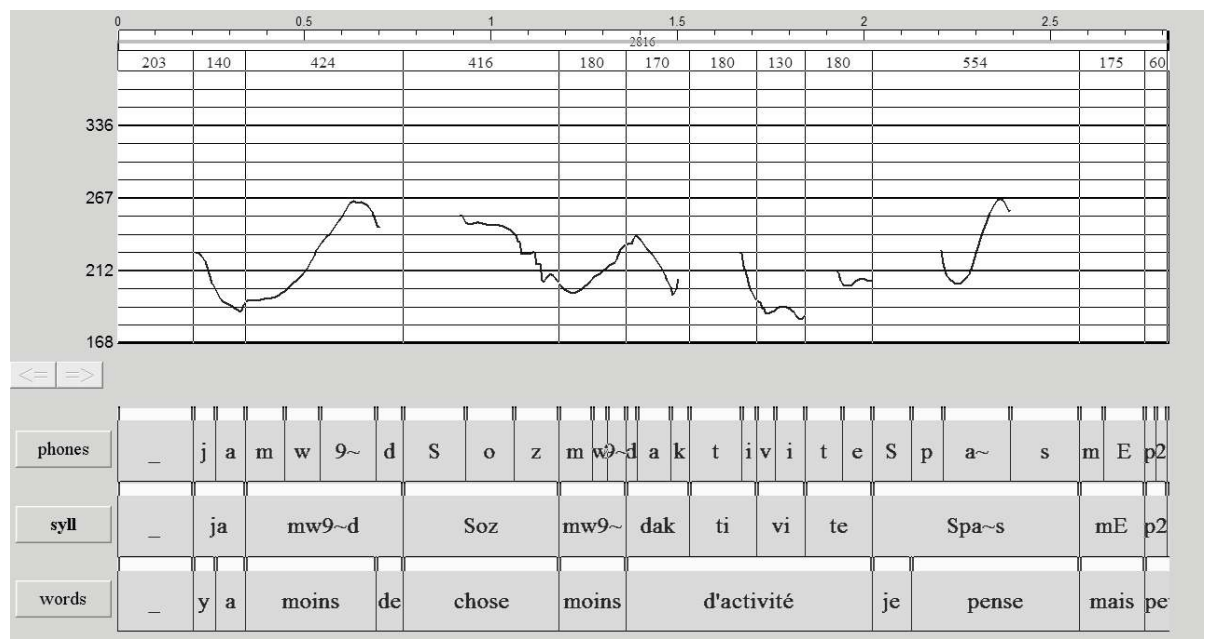

Figure 12. Copie d'écran Analor.

Tracé intonatif de l'énoncé $y$ a moins d'activités je pense [CFPP2000] 